\title{
"Memoria Abierta": patrimonio documental de organismos de derechos humanos en la Argentina
}

\author{
Graciela G. Barcala de Moyano \\ Memoria Abierta (www.memoriaabierta.org.ar)
}

\subsection{Resumen}

Presentación de los objetivos y metodología de un proyecto de preservación y organización de los archivos de ocho organizaciones no gubernamentales argentinas de defensa de los derechos humanos.

Keywords: Archivos. Organizaciones no gubernamentales. Derechos humanos. Argentina. Materiales especiales.

\subsection{Abstract}

The aims and methodology of a project for the preservation and organisation of the archives of eight Argentinian non governmental organisations in the field of human rights are presented.

Keywords: Archives. Non governmental organisations. Human rights. Argentine. Special materials.

\section{Introducción}

Este artículo relata un trabajo en ejecución - en su primera etapa (agosto 2001/octubre 2002) - denominado proyecto "Patrimonio Documental", que se desarrolla en Buenos Aires, Argentina, bajo el impulso y dirección de Memoria Abierta, una organización no gubernamental (ONG) formada por ocho organismos de Derechos Humanos. Como tarea previa al desarrollo de la exposición, a manera de introducción, en apretada síntesis y con el propósito de ubicar rápidamente en el tema, resulta oportuno explicitar algunos aspectos previos.

\section{1. ¿Qué es el Proyecto denominado "Patrimonio Documental”?}

Es uno de los cuatro proyectos que actualmente tiene en ejecución la ONG Memoria Abierta. El proyecto Patrimonio Documental ha finalizado los trabajos comprendidos en la primera etapa del mismo. Su objetivo es: 
Generar un programa coordinado de tratamiento de los archivos documentales que garantice una mayor eficacia en el uso de dicha documentación, el relevamiento, organización y preservación (1) de los documentos directamente relacionados con el período: década del 70 hasta finales de 1983 y la elaboración de un Catálogo (2) que facilite al público el acceso a la información.

La primera etapa del proyecto Patrimonio Documental se inicia en agosto de 2001 con la materialización de un relevamiento y diagnóstico realizado sobre el estado en que se encontraba el acervo documental de las organizaciones no gubernamentales que iniciaron el mismo. Este diagnóstico de los fondos documentales de los distintos organismos fue ejecutado por un equipo de trabajo reducido de Memoria Abierta y fue explicitado en un minucioso informe (Memoria Abierta, 2001). Al ser convocada por Memoria Abierta, entre diciembre de 2001 y enero de 2002, elaboré una propuesta de ordenamiento de los archivos documentales de los organismos para la aprobación por las autoridades del ente convocante. Producida la aprobación de la propuesta, a continuación se realizó la selección y la incorporación del personal a los distintos organismos participantes del proyecto para iniciar el tratamiento de la información, con el objetivo de alcanzar en octubre de 2002 el fin de la primera etapa, con la difusión en Internet del contenido del catálogo en la página web de Memoria Abierta, con un volumen aproximado de 10.000 registros de documentos.

\section{2. ¿Qué es la Organización Memoria Abierta?}

Es una ONG conformada por ocho instituciones de Derechos Humanos en la Argentina nacidas entre 1974 y 1979. Inicialmente actuaron en forma independiente, pero en octubre de 1999 comenzaron a reunirse para concretar acciones en conjunto. En marzo de 2000 se constituyeron en una alianza y celebraron un "Convenio de Cooperación" que establece el marco de trabajo para sus actividades. Seis de ellas participan del proyecto Patrimonio Documental, desarrollando actividades en sus archivos, a saber: Abuelas de Plaza de Mayo, Asamblea Permanente por los Derechos Humanos (APDH), Centro de Estudios Legales y Sociales (CELS), Familiares de Desaparecidos y Detenidos por Razones Políticas, Madres de Plaza de Mayo Línea Fundadora, y Servicio Paz y Justicia (SERPAJ). Adicionalmente, la Asociación Buena Memoria y la Fundación Memoria Histórica y Social Argentina, que no cuentan con fondos documentales propios, trabajan en la recuperación de archivos particulares.

\subsection{Los organismos que conforman la alianza: nacimiento y objetivos}

Las Abuelas de Plaza de Mayo es un organismo apartidario que surge en octubre de 1977 y tiene como objetivo localizar y restituir a los niños secuestrados desaparecidos por la represión del gobierno militar. Cuentan con un equipo

Scire. $9: 1$ (en.-jun. 2003) 139-150. 
de especialistas y un banco de datos genéticos que registra la información para identificar a todas las familiares que tienen niños desaparecidos.

La Asamblea Permanente por los Derechos Humanos (APDH), fundada en diciembre de 1975, fue el resultado de la autoconvocatoria de personalidades del quehacer nacional ante la ola de violencia y represión existente.

El Centro de Estudios Legales y Sociales (CELS) fue fundado en 1979, y entre sus objetivos están la defensa de los derechos humanos y fortalecer la democracia y el estado de derecho en Argentina. En la década de los 90 expandió sus actividades originales, y ahora se ocupa de situaciones de violencia institucional, condiciones de prisión y discriminación; y atiende al público en su moderno centro de documentación, rico en bibliografía y archivos documentales muy completos y variados.

Los Familiares de Desaparecidos y Detenidos por Razones Políticas fueron el primer grupo de afectados que funcionó en Argentina, y está constituido por padres, madres, hermanos y esposos de desaparecidos y detenidos políticos. Sus objetivos son la lucha contra la impunidad, la búsqueda de la verdad y la conservación de la memoria de los hechos ocurridos en tan doloroso pasado.

Las Madres de Plaza de Mayo nacieron en 1977, como una organización no gubernamental. Esta formada por mujeres unidas por la detención y posterior desaparición de sus hijos en manos del gobierno militar. Sus reivindicaciones son la investigación, el juicio y la condena de los responsables de estos hechos y la nulidad de las leyes de impunidad, llamadas Ley de Punto Final y Ley de Obediencia Debida. En 1986, se separan por importantes diferencias y surge la denominada Línea Fundadora.

Por último, el Servicio Paz y Justicia (SERPAJ) es una organización social que brinda solidaridad y apoya la no violencia. Fue fundada en 1974 y surge como una respuesta a hechos de injusticia. Uno de los fundadores es Adolfo Pérez Esquivel, Premio Nobel de la Paz 1980 y presidente del SERPAJ Argentina.

Los organismos mencionados, a los que se agregaron la Asociación Buena Memoria y la Fundación Memoria Histórica y Social Argentina, celebraron un convenio de cooperación en marzo de 2000. Hoy, todos ellos forman Memoria Abierta, cuyo objetivo fundamental es la preservación y sistematización de todo tipo de archivos que registren lo ocurrido en el país en el período citado, uno de cuyos proyectos es el que nos ocupa, "Patrimonio Documental.

\section{Diagnóstico sobre el estado inicial de la documentación}

El patrimonio documental de las ONG que componen Memoria Abierta, comprende material de presentación y de uso interno, testimonios, material de difusión pública, publicaciones periódicas, libros, material de prensa, material audiovisual,

Scire. $9: 1$ (en.-jun. 2003) 139-150. 
objetos de recuerdo, material para exhibiciones institucionales, informes y causas judiciales. Su proporción varía en las distintas organizaciones:

- Testimonios y denuncias: Abuelas de Plaza de Mayo - en proceso de digitalización por un equipo técnico de la Universidad Nacional de La Plata-, Asamblea Permanente por los Derechos Humanos (APDH) -6.000-, Centro de Estudios Legales y Sociales (CELS) - 370 - , Familiares de Detenidos y Desaparecidos por Razones Políticas -2.200-, Madres de Plaza de Mayo Línea Fundadora - no tienen en la sede -, Servicio Paz y Justicia (SERPAJ) - aproximadamente 400-.

- Material bibliográfico: Ordenados en Abuelas, APDH, CELS, SERPAJ.

- Material de prensa: recortes de revistas, diarios, etc. En el caso de Madres se recopilan los recortes recordatorios del Diario Página 12; y en el CELS, recortes referidos a temas de Derechos Humanos en la Argentina.

- Pancartas: Esencialmente en Madres de Plaza de Mayo Línea Fundadora.

- Producción de los organismos: boletines, revistas, libros, informes, etc. En el caso de Abuelas, se editaron libros; y para el catálogo colectivo, se analizan los sumarios.

- Otros documentos: Folletos, correspondencia, recuerdos de viajes, documentos fundacionales, material de difusión publica — solicitudes, declaraciones, invitaciones, adhesiones, volantes, afiches, etc. - , material audiovisual - videos, fotos, etc. - , objetos de recuerdo o recibidos en premio, material para muestras y exhibiciones institucionales, causas judiciales y documentos jurídicos.

Una evaluación de los fondos documentales realizada a partir de los criterios de conservación, accesibilidad e informatización revela también la diversidad de respuestas que han adoptado las distintas organizaciones, y, en definitiva, la necesidad de normalización.

\subsection{Desafíos}

El desarrollo del proyecto presenta problemas interesantes que deben ser resueltos por los profesionales que participan en el mismo. Explicitaré algunos brevemente, sin que el orden en que los trato implique prioridad alguna.

En primer lugar, los fondos documentales de los seis organismos involucrados en el Proyecto no están constituidos por libros exclusivamente, sino que la mayoría de la información está en otro tipo de documentos. Esto implica que la tarea de confeccionar la hoja de trabajo de entrada de datos antes de su ingreso a la base informatizada es difícil. El ya de por sí complejo tratamiento de la información en la biblioteca tradicional, se incrementa notablemente en este caso porque requiere una lectura detallada y completa de los textos para evaluar lo significativo y des- 
cartar lo superfluo. A esto debe agregarse que en la mayor parte de los casos se trata de documentación manuscrita, con las dificultades que ello supone.

Por otra parte, las sedes de las ONGs se encuentran en distintos lugares de la ciudad de Buenos Aires, donde se está llevando a cabo el proyecto, lo que obliga a una dispersión de los integrantes del equipo de trabajo y a un mayor esfuerzo de coordinación. Además, los horarios para el desarrollo de las tareas son diferentes en cada ONG y han dificultado la conformación del equipo de trabajo. El estado de los fondos documentales y los recursos informáticos diponibles en cada una de las ONG es muy heterogéneo. Por último, la capacitación del personal existente en cada ONG a cargo de la custodia de los fondos no es homogénea y muchas veces es inexistente para sus funciones específicas.

La cobertura temática es también muy variada: los fondos se refieren a la época fundacional de cada organismo, la Ley de Punto Final y la Ley de Obediencia Debida (4). De forma amplia y general, se analizan datos de la década del setenta hasta la actualidad. Cada organismo ha generado innumerable documentación, y hasta el momento se desconoce su volumen real y contenido. Desde mis primeras investigaciones quedó claramente en evidencia que toda esa documentación está dispersa en las organizaciones y, por lo tanto, es de difícil acceso, que se agrava por aspectos como la falta de espacios adecuados para la consulta, el hecho de que el material no esté relevado, la existencia de material documental disperso sin orden en el mismo edificio, la falta de recursos humanos idóneos, etc.

\subsection{Medidas}

Vistos los retos, elevé tres consideraciones prioritarias para ser analizadas en el marco del proyecto: la formación de un equipo idóneo y confiable, el reordenamiento físico de las colecciones, y la selección de documentación según prioridad o importancia, para garantizar la calidad de los contenidos.

Tuve también en cuenta que hasta el momento la documentación no había sido tratada por técnicos profesionales, sino archivada desde la década de los setenta. Se desconocían y aún se desconocen totalmente las características de toda ella. Al revisar con mayor detalle los archivos verticales instalados en las oficinas administrativas de algunos organismos, descubrí esquemas propios de ordenamiento del material. Éste estaba organizado por áreas temáticas que, aunque no guardaban un orden estricto, constituían elementos armoniosos por su contenido temático. Por ello, en muchos casos, tras ingresar la documentación en las bases de datos, fue almacenada finalmente en el sitio físico donde fue detectada. En otras palabras, se trató de conservar el orden preestablecido, para no alterar el procedimiento de búsqueda tradicional de los miembros de los organismos. Son las bases de datos las que permitirán recuperar todo el material existente en una primera etapa. Más adelante se contempla además la digitalizaciónn de gran

Scire. $9: 1$ (en.-jun. 2003) 139-150. 
parte de la documentación seleccionada en consulta con los representantes de los organismos.

Desde el punto estrictamente técnico, se eligió un software para registrar los datos de forma clara y concisa, y actualizar periódicamente la información de manera sencilla. Además, se contempla el acceso a los datos de los documentos a través de la página de Memoria Abierta en Internet. Ante el estado de algunas colecciones, deterioradas por la acción del tiempo y el obligado abandono en algunos casos, por situaciones de política interna, se decidió preservar la documentación (5) dentro de las normas internacionales.

Todos estos aspectos y otros que fueron surgiendo a lo largo de la primera etapa del Proyecto, tienen un único objetivo: mantener vigente la historia de hechos trascendentales de la Historia Argentina.

\section{El catalogo como unidad de información}

Para la confección de un catálogo colectivo de los seis organismos de derechos humanos, tuvimos que armonizar algunas acciones, como por ejemplo, la de gestionar la obtención periódica de la información, parte de ella guardada en otras bases de datos no compatibles con el desarrollo informático del proyecto. Para ello, se transfirieron técnicas y metodologías que permiten realizar rápidamente la integración de información de las bases de datos dispersas en una sola homogénea y apuntar a conversiones desde otros programas (6), con miras a un lenguaje de recuperación común, a través del catálogo colectivo.

Al inicio del proyecto Patrimonio Documental, se informó detalladamente a los representantes de cada organismo de los pasos a seguir y de la conveniencia de los mismos. Se requirieron - y se obtuvieron - sugerencias para concretar felizmente cada etapa. Por ejemplo, es muy interesante lo que nos pasó con las pancartas, que detallamos más delante en este trabajo, cuando surgió la necesidad de fabricar un mueble especialmente diseñado para contener esta documentación de gran volumen. Muchas Madres sugirieron diversas alternativas y pusieron mucho empeño para resolver su guarda y accesibilidad. También se analizaron con el equipo de trabajo, paso por paso, los diversos procesos técnicos, que incluyen todas las operaciones que se llevan a cabo en el esquema "Entrada, Tratamiento y Salida". Para la entrada de datos se confeccionaron hojas de acuerdo a las normas internacionales (7), específicamente las Reglas de Catalogación Angloamericanas.

Las mayores dificultades surgieron cuando se preparó la documentación jurídica, que en primera instancia se llamó "causa judicial”. En realidad, se llegó al convencimiento de que no puede ser considerada así, puesto que excepcionalmente se tiene copia de toda la causa judicial. Por lo tanto, lo más acertado, al tener

Scire. $9: 1$ (en.-jun. 2003) 139-150. 
solo una parte del todo, es denominarla "documentación jurídica". Para esta documentación jurídica, se diseñó una hoja de entrada de datos con el consenso de los abogados y bibliotecarios de aquellos organismos que poseen este tipo de documentación. La información que se incluye es el fiel reflejo de lo que se tiene en mano y también de lo que se infiere. Se citan los nombres propios - por ejemplo, represores, abogados defensores, fiscales, detenidos desaparecidos, actores - así como lugares de desaparición y/o detención - por ejemplo, provincias, ciudades, centros clandestinos de detención, testimonios, etc. Los datos que se relevan van más allá, por su complejidad, de las simples causas judiciales que habitualmente manejan nuestros colegas en las bibliotecas jurídicas.

En el caso de los testimonios, las hojas de entrada de datos constan de muchos campos que tratan de reflejar la variedad de los sucesos a relevar: lugar y fecha del testimonio, protagonismo dentro de él, otras instituciones que lo recibieron, jueces, abogados, apellidos y nombre del testigo y de las personas que se citan en su declaración, lugar de la detención, fuerza que la realizó, personas vistas en el lugar, privacidad de la denuncia, etc.

Uno de los materiales que más ha preocupado al equipo de trabajo es la correspondencia. En primer término, se seleccionó aquélla originada por cada organismo. En algunos casos, como en el de Madres de Plaza de Mayo Línea Fundadora, se trata de gran cantidad de cartas, notas, informes, circulares, artículos para revistas y recordatorios de la ONG, y, además, aquélla generada por algunas señoras a título personal, que con el correr del tiempo se ha incorporado como institucional. A simple vista sólo se trata de textos de una, dos o más hojas, pero el contenido es tan rico en connotaciones institucionales, personales y nacionales que se hace difícil no extenderse en la descripción de su contenido. Por ello, no es difícil encontrar registros con gran cantidad de datos.

Uno de los trabajos de recopilación más notorio fueron las pancartas existentes en Madres de Plaza de Mayo Línea Fundadora. El tamaño de cada una de ellas es de aproximadamente $1,20 \mathrm{~m}$ por $0,70 \mathrm{~m}$ protegidas con plásticos, según se puede observar en las marchas que tienen lugar en la ciudad. Se trata de un material documental muy rico, rara vez visto en los acervos documentales tradicionales. Contienen información de detenidos desaparecidos en la década de los setenta: fotos personales y de las familias, reseñas biográficas, reproducción de documentos personales, poesías, correspondencia, fechas de detenciones, de desapariciones y listados de personas originarios de ciudades determinadas, de colegios, de universidades, de sindicatos, listados de extranjeros, etc. Llama la atención que una cantidad notable de documentos incluidos - por ejemplo, boletines escolares, fotos, dibujos, diplomas, etc. - son originales. Las Madres se esmeran en conservar permanentemente la memoria de sus desaparecidos tanto en el mantenimiento de este material como en el de cualquier otro. Por ello, entre

Scire. $9: 1$ (en.-jun. 2003) 139-150. 
otras tareas y dentro de lo previsto, se realizaron reuniones de capacitación y divulgación con representantes de los diversos organismos.

Dada la magnitud de las tareas emprendidas y en el afán de cumplir con los plazos preestablecidos del proyecto Patrimonio Documental, se postergó para una segunda etapa el tratamiento de las innumerables colecciones de revistas. Muchas de ellas, sin duda, podrán sobrevivir al expurgo. La idea es analizarlas e incorporar información de algunos artículos seleccionados y otra documentación.

Por otro lado, se observó que muchos organismos tienen instalado el software denominado Winisis, cedido gratuitamente por la UNESCO, y muy usado en las bibliotecas y archivos del país y del exterior. En consecuencia, se resolvió trabajar primariamente con él. Mensualmente se migran los datos acumulados en un período determinado de antemano y se integran al catálogo colectivo, guardado en principio en la sede de Memoria Abierta. A partir de él, se confeccionan cada mes ocho catálogos impresos (8) con las existencias de cada organismo. En primera instancia, se los ordenó por riguroso orden alfabético de autores, con una referencia bibliográfica que identificaba el organismo de custodia, el título, la cantidad de páginas, el lugar, la fecha y los descriptores. Más adelante se decidió ordenarlos separadamente por organismo, para que cada uno de ellos pudiera consultar su propia producción y, solo si lo deseaba, la de los otros. A medida que fueron editándose mensualmente los catálogos, se fueron modificando las bases de datos, con miras a mostrar más y mejor información. Se decidió, por ejemplo, agregar el campo "nombre de personas" para incorporar el apellido y nombre de los detenidos desaparecidos, y los militares y jueces involucrados. También se hizo énfasis en el uso de zonas geográficas, indizando por provincias, ciudades, barrios, centros de detención, etc.

Por último, se está abordando el descarte, difícil por subjetivo y, no obstante, necesario, pues hay material bibliográfico fuera de contexto y una buena cantidad de duplicados. No es una tarea obligatoria, pero se debe despertar la conciencia de la necesidad de depurar y eventualmente de diseñar una política de selección atinada a aplicar en el futuro inmediato.

Los integrantes de los organismos tienen tareas muy diversas que cumplir y el tiempo siempre es escaso. Hay reuniones, convocatorias populares y actos recordatorios que les impiden en muchas ocasiones seleccionar con detalle tan amplia gama de documentación. Sin embargo, el proyecto de Memoria Abierta ha despertado en la mayoría de los organismos un interés manifiesto que se puede observar a simple vista. Evidentemente, se está en una etapa de organización, con la idea de recuperar para el futuro el pasado histórico. 


\section{Tesauro de Derechos Humanos}

Los términos controlados para la recuperación por tema de los documentos - por ejemplo, genocidio, libertad de pensamiento, peticiones, restricciones para viajar, ejecuciones extralegales, derechos del niño, etc. - se consignan en las hojas de carga de información en el campo denominado "Descriptores". Para realizar esta tarea se utilizan tanto tesauros generales como especializados en Derechos Humanos. Entre otras tareas previstas está la de confeccionar un Tesauro de Derechos Humanos al que incorporar las doscientas palabras clave que describen contenidos nuevos que no se han localizado en los tesauros especializados con los cuales se trabaja, y que son característicos de la lengua, las costumbres y la historia del país, como por ejemplo: escraches, punto final, obediencia debida, pedido de audiencia, villas miserias, violación de deberes, volante, hijos de desaparecidos, consignas, detenidos a disposición del Poder Ejecutivo Nacional (PEN), efectos psicológicos, centros clandestinos de detención, etc.

\section{Desarrollo futuro}

Esta primera etapa contempla, como se ha visto, la creación de un catálogo colectivo que refleje el patrimonio documental acumulado por las organizaciones participantes desde el período fundacional de cada una de ellos hasta la actualidad, así como su publicación en el sitio web de Memoria Abierta. En el futuro se prevé la digitalización (9) y posterior incorporación al Catálogo Colectivo, así como la catalogación de testimonios históricos, fotos y todo aquel material que aún no está totalmente relevado o permanece en casas particulares. Posteriormente, además de perfeccionar y mantener actualizadas las bases de datos y estrechar los vínculos entre las organizaciones implicadas, se prevé invitar a otras que tengan intereses comunes a integrar a este proyecto la documentación existente en ellas. $\mathrm{La}$ participación de las instituciones afines enriquecerá notablemente los contenidos y afianzará la red de información que se propone en este proyecto. Efectivamente, no existe una red cooperativa de este tipo y, sin duda, se prevé mucho interés nacional e internacional en conocer la experiencia y apoyar desarrollos futuros. En esta dirección, se está analizando la convocatoria de una reunión internacional sobre temas relacionados con el quehacer de los organismos; la preparación de publicaciones; y el rescate de la documentación existente en archivos particulares pertenecientes a las familias involucradas con los sucesos que han dado origen a este proyecto de Patrimonio Documental.

\section{Recursos humanos}

La selección de personal idóneo para la detección, selección e ingreso de información en las bases de datos fue una tarea que se realizó de forma minuciosa. Se convocaron cinco puestos para bibliotecarios y licenciados en Bibliotecología

Scire. $9: 1$ (en.-jun. 2003) 139-150. 


\section{Graciela G. Barcala de Moyano}

y Documentación y una recién egresada. Ante el desafío profesional que significa el tratamiento de documentación inédita, de alto valor histórico y sensible, no se podía dejar de advertir a los postulantes de las dificultades a las cuales se enfrentarían. El perfil convocado para realizar las tareas técnicas fue, por tanto, de especialistas con experiencia y conocimiento de gestión de la información automatizada en bibliotecas, centros de documentación y archivos: con conocimiento y experiencia en sistemas informáticos que permitan seleccionar la documentación, referenciar y automatizar los servicios; capacitados para garantizar la organización física de las colecciones por tipo de documento, ingresar información en las bases de datos, clasificar, ubicar en el estante y atender a los usuarios; con conocimientos de español e inglés; y antecedentes que garantizaran la confidencialidad y discreción que el material con el cual se trabaja requiere. Sin duda, el hecho de contar con recursos humanos idóneos para un correcto funcionamiento da los resultados esperados. También el intercambio permanente de ideas dentro del grupo de trabajo permite enfrentar las dificultades dentro de un consenso profesional. La organización de un catálogo colectivo, es una tarea que conlleva tiempo y esfuerzo y la indispensable normalización.

Las reuniones de trabajo tienen lugar una vez por mes tras la aparición del catálogo mensual en papel. Fruto de esas reuniones es la toma de decisiones técnicas, por ejemplo: la normalización de nombres institucionales, el tratamiento de las siglas, las asignación de títulos a la correspondencia, la rectificación de descriptores y la búsqueda permanentemente de la integración armoniosa de las distintas organizaciones, a través de las bases de datos correspondientes.

Por último, se debe señalar la disponibilidad de voluntarios, a los que se está capacitando para realizar labores de asistencia en esta etapa.

\section{Espacio físico}

Las autoridades de Memoria Abierta asignaron equipos informáticos y espacios físicos en su sede para las reuniones de coordinación del catálogo colectivo. Las tareas de relevamiento y tratamiento de la documentación se realizan en cada organismo. Periódicamente, se exportan los datos y se integran en la base común, para un exhaustivo control de calidad y, esencialmente, para su normalización.

La mayoría de los organismos poseen espacios, más o menos reducidos, para albergar las colecciones. En algún caso, también se usan para reuniones de los diversos sectores del propio organismo. En otros, como es el caso de la nueva sede de Madres, se destinó un espacio adecuado para uso exclusivo de biblioteca y archivo, donde se trabaja con la documentación y se la guarda ordenadamente. También en la sede renovada del CELS el espacio es amplio y confortable. 


\section{Conclusiones}

La participación de profesionales de la información para el tratamiento de la documentación en los organismos de derechos humanos argentinos constituye un precedente destacable. Hasta el momento no se habían llevado a cabo tareas de este tipo, por lo menos en Argentina, con tal nivel de complejidad y detalle. Por otra parte, uno de los mayores desafíos fue la elección de los medios organizacionales, técnicos y humanos, la integración de las tareas del equipo de profesionales - bibliotecarios, documentalistas e informáticos - y la búsqueda de las condiciones para servir de vehículo entre las autoridades de cada organismo y los técnicos. Tal como se señaló al principio, todos ellos en búsqueda de un objetivo común: seleccionar, reunir y disponer la documentación en forma sistemática, para su eventual consulta y preservar para el futuro la documentación testimonial de hechos relevantes de nuestra historia nacional.

\section{Notas}

(1) Véase Madia y Etcheverry(2002).

(2) Es factible consultar el catálogo colectivo en soporte en papel en cada organismo y a través de la página de Memoria Abierta en Internet: www.memoriaabierta.org.ar.

(4) La Ley 23492.(Ley de Punto Final) dispone la extinción de acciones penales por presunta participación en cualquier grado en los delitos del articulo 10 de la ley 23049 y en aquéllos vinculados a la instauración de formas violentas de acción política. Las excepciones y la Ley 23521 (Ley de Obediencia Debida) delimitan los alcances del deber de obediencia debida, y fijan límites al sistema de enjuiciamiento dispuesto por el artículo 10 de la Ley 23049.

(5) La prevención documental alerta sobre las amenazas que ponen en peligro las colecciones, que pueden ser de muy diferente naturaleza. Las más frecuentes — plagas, humedad, degradación ácida - son en cierta manera inevitables al formar parte del propio proceso natural de destrucción de la materia. Además, hay otros sucesos poco frecuentes -incendios, inundaciones, robos, alteración del orden público, que suelen llevar aparejadas tasas elevadas de destrucción. La luz natural y artificial decoloranlos papeles, y afecta a las tintas, fotografías, soportes magnéticos, filmes, etc. Los insectos, roedores, la temperatura y la humedad afectan definitivamente al material. El almacenamiento, la limpieza, ventilación, uso y manipulación deben ser cuidadosamente pautados para no deteriorar la documentación. En cualquier caso, la prevención y la aplicación de medidas correctoras son las soluciones más efectivas.

(6) Existen otras bases de datos - en proceso o concluidas - elaboradas con anterioridad al proyecto "Patrimonio Documental", que también se desea integrar al catálogo colectivo en la medida de las posibilidades, por ejemplo, hay bases de datos de testimonios, catálogos de libros, catálogo de fotos, etc. en disquetes, CD-ROM, videos, en otros programas, etc. Cada una de ellas recibe un análisis detallado y se resuelve individualmente en cada organismo.

Scire. $9: 1$ (en.-jun. 2003) 139-150. 
(7) "La hoja de trabajo es el medio necesario para el registro de información bibliográfica en la etapa previa a la entrada de datos al computador, en tanto no se establece un sistema de entrada directo a través de una terminal. Ella contiene las diversas áreas, campo a campo, destinadas a registrar la información sobre una unidad bibliográfica. Las hojas de trabajo del Sistema son dos: Hoja de Descripción Bibliográfica (HDB) y Hoja de Análisis de Contenido (HAC)”. (Comisión Económica para América Latina, 1984).

(8) Se considera pertinente la impresión en papel de los catálogos institucionales porque en algún caso se han extraviado las bases de datos, quedando sin acceso las colecciones.

(9) Respecto al proceso de digitalización, se estudiará la conveniencia y factibilidad de realizar la recuperación en texto completo de aquella documentación altamente relevante. Se ha propuesto y se encuentra en análisis la individualización de aquélla referida al período fundacional.

\section{Referencias}

Atchison, Jan (1989). International Thesaurus of Refugee Therminology. Dondrechd: M. Nijhoff, 1989.

Caccia, Ivana (1993). Human Rights Thesaurus. Ottawa, University of Ottawa, 1993.

Reglas de Catalogación Angloamericanas / preparadas bajo la dirección del Joint Steering Committee for Revisión of AACR, un Comité de la American Library Association. $2^{\mathrm{a}}$ ed. rev. 1988, enmiendas 1993 y 1997. Santafe de Bogotá: Rojas Eberhard Editores, 1998. $764 \mathrm{p}$.

Stormorken, Bjorn; Zwaak, Leo (1988). Human rights terminology in international law: a thesaurus. Boston: M. Nijhoff; Strasbour: Council of Europe, Human Rights Documentation Centre, 1988. 234 p.

Comisión Económica para América Latina. Sistema de información bibliográfica: uso de hojas de trabajo (HDB y HAC) y tarjeta de registro bibliográfico (TRB). Santiago de Chile: Naciones Unidas, 1984.

Comisión para la Defensa de los Derechos Humanos en Centroamérica (1987). Tesauro Centroamericano de Derechos Humanos. CODEHUCA-CSUCA: San José de Costa Rica: 1987. 169 p.

Madia, M. Eugenia; Etcheverry, Lilia (2002). Conservación preventiva: resumen. Buenos Aires: julio 2002.

Memoria Abierta (2001). Diagnóstico de los Fondos Documentales de los Organismos de Derechos Humanos. Buenos Aires, 3 de octubre de 2001.

Naciones Unidas (1986). UNBIS Tesauro. Edición española. Nueva York, Biblioteca Dag Hammarskjold. Naciones Unidas, 1986.

UNESCO (1995). Unesco Thesaurus = Thesaurus de l'Unesco = Tesauro de la UNESCO. Paris: 1995.

Valdez, Patricia (2000). La Construcción de la Memoria sobre el pasado autoritario en Argentina [Comunicación a congreso]. // The Latin American Studies Association. XXII Congreso Internacional, Miami, Florida, 16 al 18 de marzo de 2000. Patricia Valdez es consultora independiente, directora de Memoria Abierta y miembro de la Comisión Directiva del Centro de Estudios Legales y Sociales (CELS).

Scire. $9: 1$ (en.-jun. 2003) 139-150. 